\title{
Late Diagnosis among patients with Prostate Cancer at the Uganda Cancer Institute: A retrospective cohort study.
}

Nelson Bunani ( $\nabla$ n.bunani@yahoo.com )

Makerere university school of public Health https://orcid.org/0000-0001-5367-2175

Angela Nakanwagi Kisakye

Makerere University School of Public Health

Aloysius Ssennyonjo

Makerere University School of Public Health

Fred Nuwaha

Makerere University School of Public Health

Research article

Keywords: Diagnosis, Prostate cancer, Uganda, Timing, late diagnosis.

Posted Date: February 3rd, 2020

DOI: https://doi.org/10.21203/rs.2.11766/v3

License: (c) (i) This work is licensed under a Creative Commons Attribution 4.0 International License.

Read Full License 


\section{Abstract}

Background Late diagnosis of prostate cancer is common in Uganda and elsewhere. Diagnosis in advanced stages is associated with high mortality, morbidity and low quality of life. We estimated time taken from perception of symptoms attributable to prostate cancer to biopsy among patients with prostate cancer at the Uganda Cancer Institute $(\mathrm{UCl})$ and the associated factors. Methods We conducted a retrospective cohort analysis of records of 280 patients with histologically confirmed diagnosis of prostate cancer at UCI from January 2016 to December 2017. Time to diagnosis was obtained from the difference between approximate date of onset of initial symptoms and date when a biopsy was taken. Late diagnosis was that when an individual was diagnosed with prostate cancer stage III or IV whereas stages I and II were classified as early. We used modified Poisson regression to assess factors associated with timing of diagnosis among the patients. Results The median time from first perceived symptoms to biopsy for prostate cancer patients was 12 (IQR 5-24) months and 76\% were diagnosed after 4 months of

symptoms. Median age at time of diagnosis of patients was 70 (IQR66-74.5) years and at least $50 \%$ were aged between $65-74$ years. About $81.8 \%$ of patients were diagnosed late; of which $35.7 \%$ were in stage III and $46.1 \%$ were in stage IV. Nearly all patients presented with raised prostate specific antigen with a median prostate specific antigen of 100.2 (IQR $36.02-350) \mathrm{ng} / \mathrm{ml}$ of blood at the time of admission. In adjusted analysis, patients whose biopsies were taken before 5 months of recognising symptoms were two times as likely to have cancer stage I and II compared to those patients in whom the biopsies were taken after 4 months. Conclusion More than three in four patients were diagnosed late. Taking a biopsy after 4 months of initiation of symptoms was partially responsible for the delay. To improve time to diagnosis, communities should be educated about symptoms of prostate cancer and advised to seek health care early. Health care workers should be sensitised to suspect prostate cancer among patients to allow timely referral for specialised assessment and management.

\section{Background}

Prostate cancer is one of the big public health problems globally and is the third commonest cancer diagnosed worldwide responsible for $7.1 \%$ of all cancer deaths (1). Prostate cancer is the second most diagnosed malignancy and the second-leading cause of cancer-related deaths among men globally (2). The burden of prostate cancer is relatively higher in sub-Saharan Africa where it accounts for $20.3 \%$ of all cases of cancer in men with the highest incidence rate reported in Zimbabwe and Uganda $(3,4)$.

In Uganda prostate cancer has been increasing at a rate of $5.2 \%$ annually, making it the most rapidly increasing cancer in the country and in sub-Saharan Africa (5). Despite the increase in incidence, approximately $90 \%$ of prostate cancer patients are not aware of the disease and as a result they do not take early urinary symptoms seriously hence they are diagnosed late with very advanced disease in stage IV presenting with incurable tumors (5-7). Late diagnosis limits treatment options, increases mortality and leads to low quality of life for patients and their families $(8,9)$. The factors associated with late diagnosis and consequently late treatment have not been well evaluated especially in low income countries of sub-Saharan Africa. 
Previous studies in Uganda found the median age of prostate cancer diagnosis to be 70 years among Ugandan men with majority dying within the first year of diagnosis and only $46.9 \%$ living 5 years after diagnosis (10). Early diagnosis of prostate cancer may lead to timely management of the disease which can lead to better treatment outcomes. We conducted this study to estimate the timing of diagnosis among prostate cancer patients in Uganda and the associated factors.

\section{Methods}

\section{Aim, design and setting}

We conducted this study to generate information on the timing of diagnosis and associated factors among prostate cancer patients in Uganda. We hope that this study will provide evidence for health care providers and policy makers on the need to design programs that will improve the timing of diagnosis of prostate cancer. This was a retrospective cohort study in which we quantitatively analyzed patient records of men with a histologically confirmed diagnosis of prostate cancer at UCI between January 2016 and December 2017.

We conducted the study at the Uganda Cancer Institute (UCI). $\mathrm{UCl}$ is a public specialized tertiary care medical facility owned by the Government of Uganda. It is part of Mulago National referral hospital complex located along Upper Mulago Hill Road, on Mulago Hill about five kilometers from Kampala central business district. The institute has an inpatient facility with a capacity of about 80 beds and receives an average of about 200 out patients daily.

Specialized treatment for prostate cancer in Uganda is only offered at UCl. All suspected and confirmed prostate cancer patients in the country are supposed to be referred to $\mathrm{UCl}$ for management. The guidelines for diagnosis and management of patients with suspected cancer of the prostate cover both symptomatic and asymptomatic cases. The guidelines for patients with symptoms state that prostate cancer screening should be considered in any male above 50 years of age and or above 40 years of age if he has a first degree relative who has or had prostate cancer and or is of African ethnicity. For asymptomatic men seeking screening, the guidelines recommend counselling to undertake a prostate specific antigen (PSA) test and digital rectal exam (DRE) if PSA is raised. Referral to UCl should be considered after two abnormal PSA at least 6 weeks apart and or abnormal hard prostate on DRE (11). Prostate cancer staging at UCl is done clinically using DRE, results of PSA and Gleason score.

\section{Description of Materials}

We abstracted data from records of all newly diagnosed prostate cancer patients registered at $\mathrm{UCl}$ from January 2016 to December 2017. These records included; patient files, registers, summary sheets, doctors' medical notes and referral notes. The data was abstracted using a data abstraction tool. All patients reported symptoms at the time of diagnosis which included frequent micturition, weak flow of urine, urge to urinate frequently at night, blood in the urine, erectile dysfunction and burning sensation 
during urination. We excluded 15 patient records which did not indicate the date of onset of the initial perceived symptoms and date when diagnosis was made.

\section{Study variables}

The primary outcome variable was stage of prostate cancer at the time of diagnosis. Stages 1 and 2 were classified as early, whereas stages 3 and 4 were classified as late. This classification was based on prostate cancer staging by American cancer society (12). Independent variables included time taken to diagnosis, age, family history of prostate cancer, education level, occupation, ethnicity, marital status, religion, co-morbidities and presenting symptoms. The time to diagnosis was obtained from the difference between the recorded estimated time of onset of initial symptoms and the time when a biopsy was done.

\section{Data management}

The data were collected, cleaned and entered into Microsoft Excel 2016. Continuous numerical responses were entered as absolute values while categorical responses were coded. The data was stored in a confidential manner in a password-protected computer.

\section{Data analysis}

Data were analyzed using STATA version 14. All continuous variables were summarized using medians with interquartile ranges while categorical data were recorded as proportions with percentages. We used Pearson's Chi-square test to examine the associations between independent variables and timing of diagnosis among prostate cancer patients. Modified Poisson regression with robust variances was used at bivariable and multivariable analysis to identify factors associated with timing of diagnosis among prostate cancer patients at UCl. Prevalence ratios (PRs) were used to estimate the strength of association between the outcome and indicator variables and associations were tested at a $95 \%$ confidence interval $(\mathrm{Cl})$.

\section{Results}

The median time to diagnosis for the prostate cancer patients at UCI between January 2016 and December 2017 was 12 (IQR5-24) months. Only $24 \%$ of the patients were diagnosed with in the first four months of perception of the symptoms. Their median time to admission at $\mathrm{UCl}$ from the initiation of symptoms was 14 (IQR 6-24) months. More than three quarters (81.8\%) of the patients were diagnosed late, of which $35.7 \%$ were in stage III and $46.1 \%$ were in stage IV (table 1 ).

\section{Table 1: Timing of diagnosis and histo-pathological findings of the prostate cancer patients diagnosed at Uganda Cancer Institute, January 2016 and December 2017}




\begin{tabular}{|c|c|c|c|c|}
\hline Diagnosis \& Histopathological findings & Frequency & $\%$ & Median & IQR \\
\hline Time to diagnosis from first perceived symptoms & & & 12 & $5-24$ \\
\hline 1-4 months & 68 & 24.0 & & \\
\hline $5+$ months & 212 & 75.7 & & \\
\hline \multicolumn{5}{|l|}{ Cancer stage } \\
\hline 1 & 41 & 14.6 & & \\
\hline 2 & 10 & 3.6 & & \\
\hline 3 & 100 & 35.7 & & \\
\hline 4 & 129 & 46.1 & & \\
\hline \multicolumn{5}{|l|}{ Gleason score } \\
\hline$\leq 6$ & 41 & 14.6 & & \\
\hline 7 & 10 & 3.6 & & \\
\hline 8 & 98 & 35.0 & & \\
\hline \multirow[t]{2}{*}{9} & 76 & 27.1 & & \\
\hline & 55 & 19.6 & & \\
\hline 10 & & & & \\
\hline
\end{tabular}

Overall, prostate cancer patients presented with very high PSA levels. Their median age at the time of diagnosis was 70 (IQR 66-74.5) years. Eighty three percent of these patients were married and at least $85 \%$ had other comorbidities which included hypertension, diabetes, ulcers, liver disease, and urinary issues. Catholics constituted 129 (47.3\%) of the patients and most of the patients 197(78.5\%) had no family history of prostate cancer. Less than half of the patients $113(40.4 \%)$ were Baganda and they were mainly peasant farmers. Only $24 \%$ of the diagnosed patients had a digital rectal exam done as a way of testing for prostate cancer. Most patients, 176 (64.5\%) presented mainly with lower urinary symptoms. These included frequent micturition, weak flow of urine, urge to urinate frequently at night, blood in the urine, erectile dysfunction and burning sensation during urination. At the end of the 2 years $63.2 \%$ of the patients had been lost to follow up, $12.1 \%$ were still in care and $24.7 \%$ had died. More than three quarters of the patients $273(86.2 \%)$ had metastases which were mainly in the bones, lymph nodes, rectum, liver, spine and the bladder.

Table 2: Characteristics of prostate cancer patients diagnosed between January 2016 and December 2017 at the Uganda Cancer Institute 


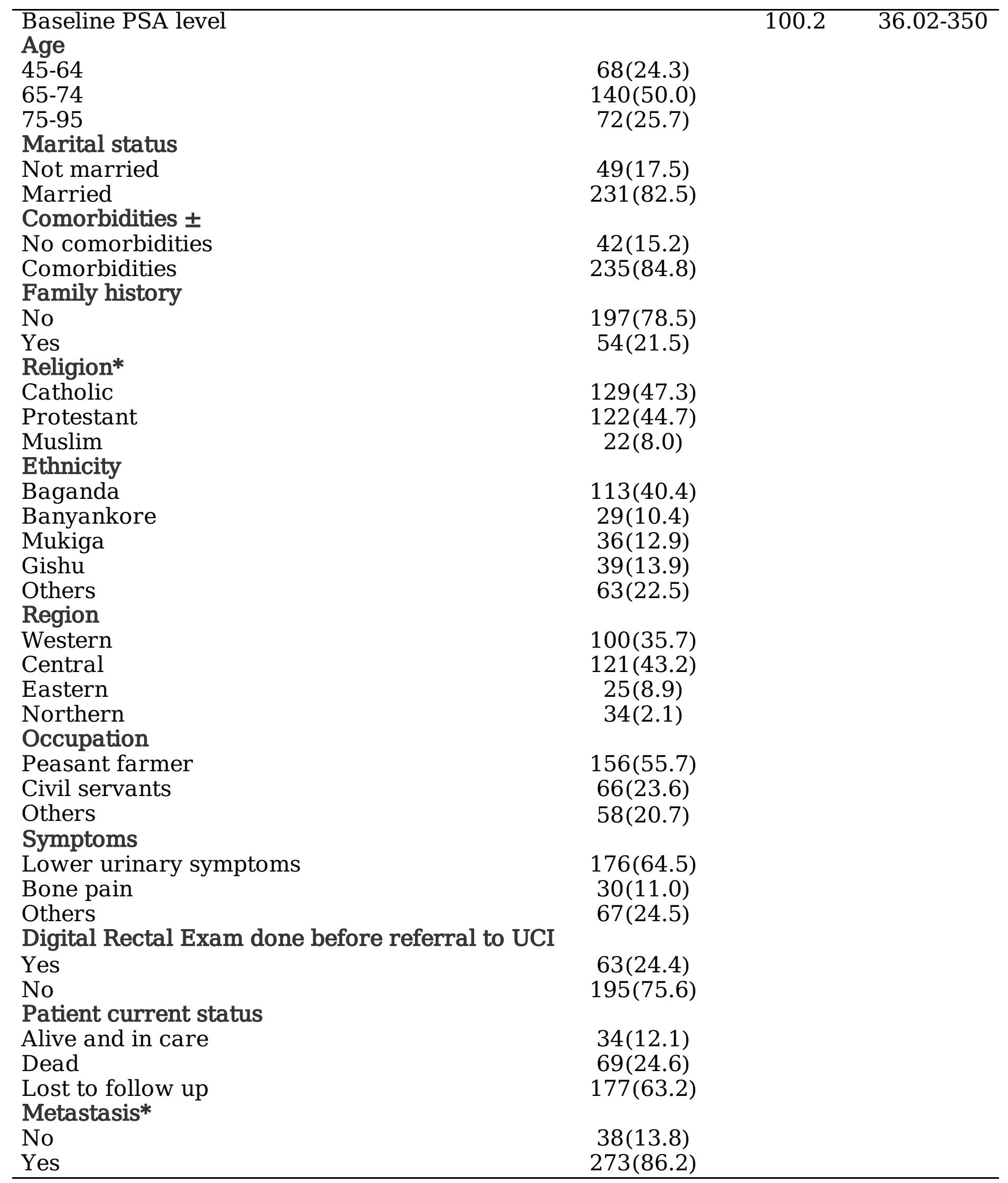


* Variables that had missing data.

Most of the symptoms were low urinary. Digital rectal was done in some health facilities that provide prostate cancer screening and UCI. None of the patients was asymptomatic at the time of diagnosis.

\pm comorbidities included hypertension in 106 (37.9\%) of patients, diabetes mellitus in 15 (5.4\%) of patients, Urinary tract infections in 62 (22.1\%) of patients and Kidney disease in 14 (5.0\%).

Other symptoms include blood in urine, erectile dysfunction, general body weakness, fever, malaise and loss of weight.

Areas of metastasis included; bones, lymph nodes, rectum, liver, spine and the bladder.

At bivariable analysis, only the time taken to request biopsy from the time first symptoms appeared was significantly associated with timing of diagnosis with P-value 0.012 .

Table 3: Bivariable and Multivariable analysis of the factors associated with timing of diagnosis among patients with prostate cancer at UCI. 


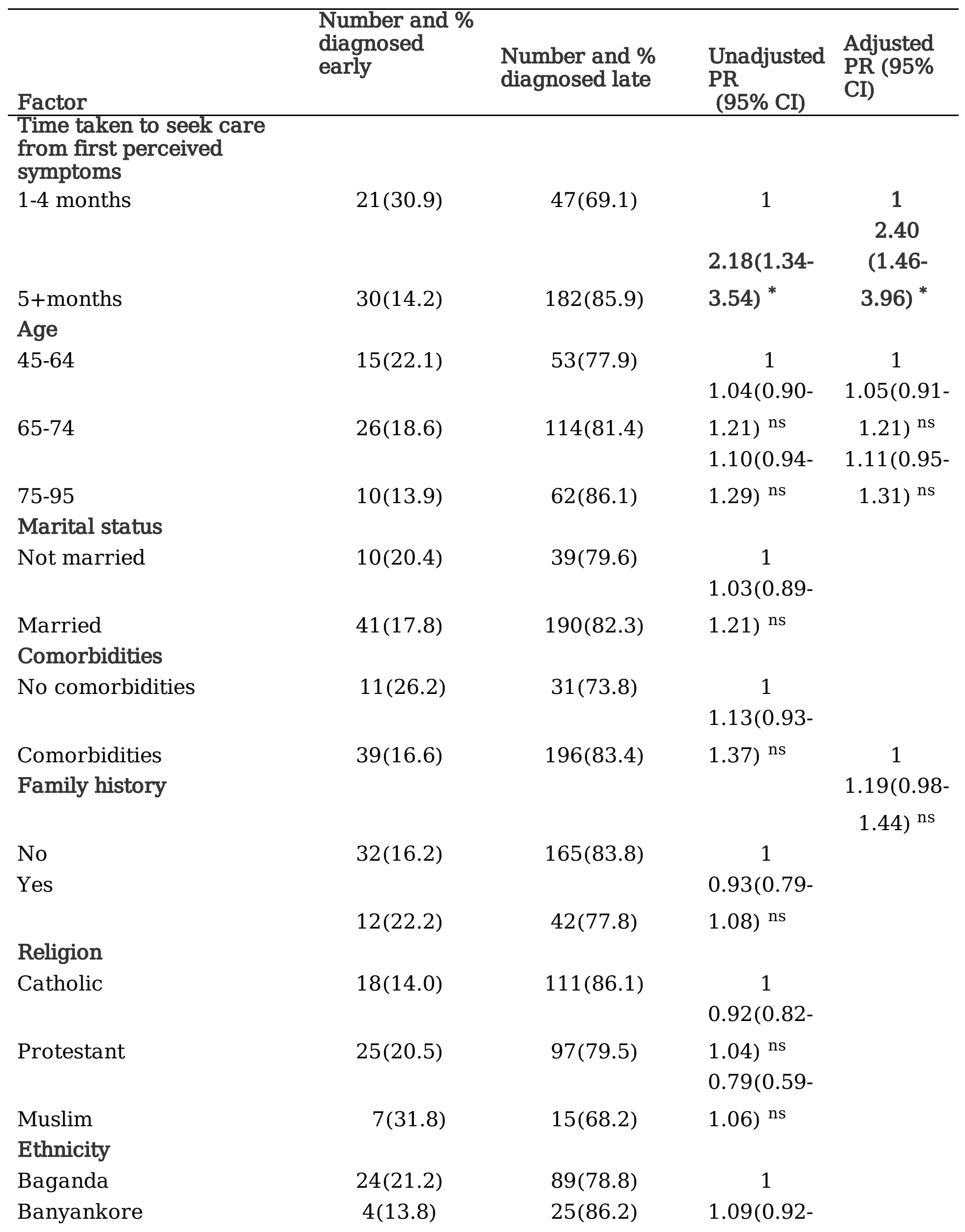


Bagishu

Bakiga

Others

Region

Western

Central

Eastern

Northern

Occupation

Peasant farmer

Civil servants

$$
9(25)
$$

$$
\text { 4(10.3) }
$$

10(15.9)

17(17.0)

24(19.8)

3(12.0)

7(20.6)

26(16.7)

13(19.7)
1.30) $\mathrm{ns}$

0.95(0.77-

1.18) ${ }^{\mathrm{ns}}$

$1.14(0.99$ -

35(89.7)

$1.31)^{\mathrm{ns}}$

1.07(0.92-

53(84.1)

$1.23)^{\mathrm{ns}}$
83(83.3) 1

0.97(0.85-

97(80.2) 1.10) ${ }^{\mathrm{ns}}$

1.06(0.89-

22(88)

$1.26)^{\mathrm{ns}}$

$0.96(0.79$ -

27(79.4)

1.16) ${ }^{\mathrm{ns}}$

$130(83.3)$

1

0.96(0.84-

17.86

53(80.3)

$1.11)^{\mathrm{ns}}$

0.95(0.82-

Others

12(20.7)

46(79.3)

$1.10)^{\mathrm{ns}}$

Digital Rectal Exam done *

Yes

8(12.7)

No

\section{Presenting symptoms}

Lower urinary symptoms

Bone pain

38(19.5)

$29(16.5) \quad 147(83.5)$

4(13.3)

55(87.3)

$0.92(0.82-$

$1.04)^{\mathrm{ns}}$

$0.87(0.79$ -

157(80.5)

$0.96)^{\mathrm{ns}}$

Others

17(25.4)

26(86.7))

$50(74.6)$

${ }^{*} \mathrm{P}<0.05,{ }^{\mathrm{ns}}$ : not statistically significant 
At multivariable level, biopsies made/taken within 4 months of recognizing symptoms was associated with early or late diagnosis among patients with prostate cancer. After controlling for age, comorbidities, religion and presenting symptoms; the chance of being diagnosed early was 2.04 times among patients who had a prostate biopsy within 4 months diagnosed from the time they felt the initial symptoms compared to those who had a biopsy after four months from the time they felt the first symptoms (adjusted PR 2.40, 95\% $\mathrm{Cl} 1.46-3.96, \mathrm{P}=0.001$ ).

\section{Discussion}

We found that the median time to diagnosis for prostate cancer patients was 12 months from the time the patients recognized symptoms that may have been attributable to cancer of the prostate. This time was slightly higher than what was reported in Burkina Faso where the prostate cancer patients spent an average of 11 months to diagnosis (13). Most of the patients were diagnosed late with metastatic cancer and very high levels of prostate specific antigen (PSA). Patients who had biopsies within 4 months of initiation of symptoms were two times likely to be diagnosed early compared to patients who had biopsies after 4 months. This is comparable to a studies conducted in Florida, USA and Toronto, Canada which found that men diagnosed with early stage prostate cancer had a shorter wait time to diagnosis (14).

Late diagnosis could have been due to patients' delay in seeking health care for the symptoms, and or due to health systems delays in requesting and performing of necessary investigations including prostate biopsies. The possible causes of patient delay include lack of knowledge regarding importance of symptoms, trivialization of symptoms, financial and economic barriers, poor health seeking behavior for men, lack of trust in health systems and seeking care from non-professionals such as traditional healers, spiritual healers and Chinese herbalists $(15,16)$. On the other hand, health system delays may be attributable to inadequate number of health care workers able to attend to patients, lack of knowledge and skills to assess patients, limited diagnostic equipment as well as lack of supplies, delayed referrals, and health workers not considering cancer of prostate in their differential diagnosis. Related studies in low and middle income countries also found an association between delays and late diagnosis among cancer patients $(16,17)$. Such late diagnosis results into poor prognosis and death $(18)$.

Related studies in Uganda and other developing countries have found other possible causes of patient and health system delays to include patients non-adherence to the recommended investigations, referral complexity as cancer investigations are done from different locations and lack of large scale populationbased prostate cancer screening $(5,10,19)$. Indeed, in our cohort all patients were assessed for prostate cancer after developing symptoms. No wonder then that most of them presented with late diagnosis. Even among patients in whom biopsy was done within 4 months of symptoms, $69 \%$ had advanced disease. 
We further found that most patients were lost to follow up while others had succumbed to prostate cancer within two years. The high loss to follow-up may be related to limited capacity of UCI to provide adequate care and follow-up due to limited staffing levels (20). This implies that the health system in Uganda and the $\mathrm{UCl}$ need to do more follow up of patients after confirmatory diagnosis of prostate cancer to improve prognosis of prostate cancer patients.

\section{Strength and limitations}

The major strength of this study was that it was conducted in a national referral facility which is the only center that provides cancer treatment in Uganda, this helped us to capture data from all the patients across Uganda.

This study neither differentiated between patient and health system delays nor analyzed the reasons for the delays. Future studies are needed to differentiate between patient and health system delays and to elucidate the reasons for these delays. Furthermore, there could have been some confounding by indication of biopsy due to attribution of lower urinary symptoms to an enlarged prostate rather than cancer that could have led to an earlier biopsy and earlier diagnosis especially when digital rectal examinations are rarely done.

\section{Conclusion And Implications}

Most patients with prostate cancer were diagnosed late. Patients who had a biopsy within 4 months of initiation of symptoms had a higher chance of being diagnosed early. Our data suggest that the community should be educated about symptoms of prostate cancer and advised to seek health care early. In addition, the health care system should be strengthened to detect and diagnose prostate cancer in a timely manner. For instance, health care workers should be educated to suspect prostate cancer among their patients so that they are able to refer such patients for appropriate specialized assessment and management. More research is needed to better understand reasons for delays and to evaluate interventions aimed at reducing the delay in diagnosis of prostate cancer (e.g.) regarding the feasibility of screening asymptomatic men.

\section{List Of Abbreviations}




\begin{tabular}{ll} 
UCI & Uganda Cancer Institute \\
\hline PSA & Prostate Specific Antigen \\
\hline IRB & Institutional Review Board \\
\hline HDREC & Higher Degrees Research and Ethics Committee \\
\hline DRE & Digital Rectal Exam \\
\hline UBOS & Uganda Bureau of Statistics \\
\hline MOH & Ministry of Health
\end{tabular}

\section{Declarations}

\section{Ethics approval and consent to participate}

We obtained ethical approval to conduct the study from Makerere University School of Public Health Higher Degrees Research and Ethics Committee (HDREC) and Uganda Cancer institute Institutional Review Board (IRB) (Ethics certificate number REO/AC/002)

Patient files were identified using the prostate cancer patient registration numbers and no personal identifiers were used. We handled all the data that we collected and the patient files with confidentiality.

\section{Consent for publication}

Not applicable

\section{Availability of data and material}

The datasets used and/or analyzed during the study are available from the corresponding author upon reasonable request.

\section{Competing interests}

The authors declare that they have no competing interests.

\section{Funding}

This work was solely funded by the corresponding author as a requirement for the Master of Public Health dissertation.

\section{Authors' contributions}

NB, AS and FN contributed towards the study design. NB contributed towards literature search, data analysis and drafting of first version of manuscript. ANK contributed towards reviewing the initial drafts 
of the manuscript. All authors contributed towards data interpretation and critical comments on the first and subsequent drafts of the manuscript. All authors read and approved the final manuscript.

\section{Acknowledgements}

The authors would like to thank, Makerere University School of Public Health and the Uganda Cancer Institute. The authors would also like to thank the African Field Epidemiology Network (AFENET) for the support that was offered through the AFENET Scientific Writer, Ms. Angela Kisakye. This work forms part of a Master's degree for one of the authors (Nelson Bunani), with Makerere University, Kampala Uganda.

\section{References}

1. Bray F, Ferlay J, Soerjomataram I, Siegel RL, Torre LA, Jemal A. Global cancer statistics 2018: GLOBOCAN estimates of incidence and mortality worldwide for 36 cancers in 185 countries. CA Cancer J Clin. 2018;68(6):394-424.

2. Tourville EA, Nguyen MM. Prostate cancer detection by using digital rectal examination: contemporary practice patterns in the United States. Clin Genitourin Cancer. 2013;11(3):263-269.

3. Parkin DM, Bray F, Ferlay J, Jemal A. Cancer in africa 2012. Cancer Epidemiol Prev Biomark. 2014;23(6):953-966.

4. Chu LW, Ritchey J, Devesa SS, Quraishi SM, Zhang H, Hsing AW. Prostate cancer incidence rates in Africa. Prostate Cancer. 2011;2011.

5. Okuku F, Orem J, Holoya G, De Boer C, Thompson CL, Cooney MM. Prostate Cancer Burden at the Uganda Cancer Institute. J Glob Oncol. 2016;2(4):181-185.

6. Nakandi H, Kirabo M, Semugabo C, Kittengo A, Kitayimbwa P, Kalungi S, et al. Knowledge, attitudes and practices of Ugandan men regarding prostate cancer. Afr J Urol. 2013;19(4):165-170.

7. Busingye G. Knowledge, Attitude and Practice of Men Aged 40-75 Years Towards Prostrate Cancer Screening in Nakawa Division Kampala District. [PhD Thesis]. International Health Sciences University.; 2015.

8. Dale W, Bilir P, Han M, Meltzer D. The role of anxiety in prostate carcinoma: a structured review of the literature. Cancer Interdiscip Int J Am Cancer Soc. 2005;104(3):467-478.

9. Samuels M, Pottage C, Dai D MP. Waiting Times for Suspected and Diagnosed Cancer Patients 2015-16 Annual Report. Lond UK NHS. 2016;

10. Gondos A, Brenner H, Wabinga H, Parkin DM. Cancer survival in Kampala, Uganda. Br J Cancer. 2005;92(9):1808.

11. Uganda Cancer Institute. Referral Guidelines for suspected Cancer [Internet]. Kampala; 2016 [cited 2019 Jun 26]. Available from: https://www.uci.or.ug/download/publications/

12. American Cancer society. Prostate Cancer Early Detection, Diagnosis, and Staging [Internet]. 2016. Available from: https://www.cancer.org/content/dam/CRC/PDF/Public/8795.00.pdf 
13. Kaboré FA, Zango B, Kambou T, Ouédraogo AS, Bambara A, Yaméogo C, et al. Prostate Cancer Disease Characteristics at the Time of Diagnosis and Initial Treatment Offered in a Tertiary Hospital at Ouagadougou (Burkina Faso). Open J Urol. 2014;4(01):7.

14. Stevens C, Bondy SJ, Loblaw DA. Wait times in prostate cancer diagnosis and radiation treatment. Can Urol Assoc J. 2010;4(4):243.

15. Otieno ES, Micheni JN, Kimende SK, Mutai KK. Delayed presentation of breast cancer patients. East Afr Med J. 2010;87(4):147-150.

16. Pace LE, Mpunga T, Hategekimana V, Dusengimana J-MV, Habineza H, Bigirimana JB, et al. Delays in breast cancer presentation and diagnosis at two rural cancer referral centers in Rwanda. The oncologist. 2015;20(7):780-788.

17. Ferlay J, Shin H-R, Bray F, Forman D, Mathers C, Parkin DM. Estimates of worldwide burden of cancer in 2008: GLOBOCAN 2008. Int J Cancer. 2010;127(12):2893-2917.

18. Brinton LA, Figueroa JD, Awuah B, Yarney J, Wiafe S, Wood SN, et al. Breast cancer in Sub-Saharan Africa: opportunities for prevention. Breast Cancer Res Treat. 2014;144(3):467-478.

19. Ikuerowo SO, Omisanjo OA, Bioku MJ, Ajala MO, Mordi VPN, Esho JO. Prevalence and characteristics of prostate cancer among participants of a communitybased screening in Nigeria using serum prostate specific antigen and digital rectal examination. Pan Afr Med J. 2013;15(1).

20. Government of Uganda. UBOS. Uganda Bureau of statistics, Statistical Abstract 2018 [Internet]. 2018 [cited 2019 Jun 26]. Available from: https://bit.ly/2FAHyJQ 